\title{
Journal of Pharma and Pharmaceutical Sciences
}

\section{Statutory Imposed Terms of Stress, Distress, Well-Being and Animal Welfare: Suggested Guidelines for Humane Endpoints in Animal Studies}

\author{
David V. Gauvin*, Lisa Craig and Scott E. Boley
}

Drug Safety Evaluation, MPI Research, Mattawan, MI, USA

\begin{abstract}
*Corresponding author: David V. Gauvin, Drug Safety Evaluation, MPI Research, Mattawan, MI, USA; Email: David.Gauvin@mpiresearch. com
\end{abstract}

Article Type: Review, Submission Date: 08 February 2018, Accepted Date: 05 March 2018, Published Date: 22 March 2018.

Citation: David V. Gauvin, Lisa Craig and Scott E. Boley (2018) Statutory Imposed Terms of Stress, Distress, Well-Being and Animal Welfare: Suggested Guidelines for Humane Endpoints in Animal Studies. J.Pharm Pharm Scien 2(2): 1-13. doi: https://doi.org/10.24218/ vjpps.2018.21.

Copyright: @ 2018 David V. Gauvin, et al. This is an open-access article distributed under the terms of the Creative Commons Attribution License, which permits unrestricted use, distribution, and reproduction in any medium, provided the original author and source are credited.

\section{Abstract}

Bad terminology is the enemy of good science. The US Congress passed the "Plain Writing Act of 2010" that required all federal agencies to use clear and concise language that the general public can understand and use. However, the Animal Welfare Act [1] and Good Laboratory Practice Guidelines [2] were written well before the enactment of the Plain Writing Act.The Study Director (SD) must strike a balance between the best interests of the client, contract research organization (CRO), veterinary staff, regulatory reviewers, as well as the animals in an effort to comply with guidance documents and regulations that are filled with poorly-defined terminology.Poorly chosen, legally ill-defined terms such as "well-being", "welfare", "distress", and "anxiety" lead to confusion and discord during the nonclinical development of a drug.US civil courts have been struggling with these terms for years when defining damages in human personal injury cases; so it is not surprising the level of uncertainty that exists when applying these terms to animal research. Science is value-laden: it values ethical standards of conduct, objectively verifiable and legally defensible data, as well as clear and concise language in reporting the results. Three "seasoned" Study Directors with cumulative regulatory research history of $>60$ years and cumulative experiences on an Institutional Animal Care and Use Committee (IACUC) of $>20$ years provide a view of the current research climate and propose some sound, legally-defensible processes in this balancing act required for drug development.

Keywords: Animal Welfare, Institutional Animal Care and Use Committee, IACUC, Animal distress, Stress, Animal Welfare Act, Well-being, Humane endpoints, Euthanasia.

\section{Introduction}

Three separate acts of Congress set into motion a quaqmire of institutional conflicts between government agencies and the pharmaceutical industry. In enacting the Food, Drug \& Cosmetic
Act, the US Congress set a key priority for the Food and Drug Administration (FDA) to improve the safety and effectiveness of medical products, both through rigorous review of nonclinical and clinical studies and manufacturing process information before products are approved, as well as through monitoring actual patient experiences and manufacturing quality once the products are on the market. FDA aims to fulfill its public health mission by setting clear standards and guidelines for evaluating the safety and effectiveness of all new human and veterinary drug products so that the regulatory process can keep pace with advances in science and technology. In 1978, through administrative action, the FDA set forth the Good Laboratory Practice Regulations for Nonclinical Laboratory Studies [2] which are designed to assure high quality testing required to evaluate the safety of regulated products in the U.S. The GLPs required the test facility management of each research facility to identify and place a single person as a central point of control for each safety assessment study conducted as part of submission of a new drug application - the Study Director (SD; [2] \$58.33, p. 60015). The SD has the overall responsibility for the technical conduct of the study and it was the stated intent of the FDA that the SD was the individual responsible for the entire study, which included the overall conduct including animal care (p 59992).

The second major piece of legislation influencing the conduct of animal research by Congress established a regulatory scheme for the Department of Agriculture to adopt statutory and administrative powers over the transportation, sale, and handling of certain animals ([1] 7 USC $\$ 2131$ - 2159; 18 USC $\$$ 49). This was intended to regulate who may possess or sell certain animals as well as the living conditions (for non-agricultural, domestic animals) under which the animals must be kept. Congress defined the primary activity of the USDA in bona fide research including the registration of certain animal users and the requisite inspections of the facilities of those users to 
Citation: David V. Gauvin, Lisa Craig and Scott E. Boley (2018) Statutory Imposed Terms of Stress, Distress, Well-Being and Animal Welfare: Suggested Guidelines for Humane Endpoints in Animal Studies. J.Pharm Pharm Scien 2(2): 1-13. doi: https://doi.org/10.24218/vjpps.2018.21.

determine whether the care guidelines or regulations for animals in their possession are being followed. This is most commonly referred to as the "Animal Welfare Act" (AWA). It is extremely troublesome within the pharmaceutical industry and research academia that in passing the AWA Congress injected confusion and debate into the nonclinical safety process by inserting the term "psychological well-being" into the law books without a statutory (legal) definition. A myriad of views and definitions have been used in the discussion of stress and distress. While there is little denial on either side of the veterinary intervention argument that stress is a real or perceived threat to an animal's physiological homeostasis, defining "animal well-being" without relying on some form of anthropomorphic measures is a challenge to both regulators and researchers.

In 2010, Congress passed the "Plain Writing Act" [3] to improve the effectiveness and accountability of Federal agencies to the public by promoting clear government communication that the public can understand and use. As described by the FDA, government agencies such as the FDA should issue communication that helps the targeted audience to:

1. Easily find what is needed,

2. Understand what is heard or read, and,

3. Use it the first time it is read or heard ( https://www.fda.gov/ aboutfda/plainlanguage/ ).

It should be highlighted that both criminal and civil courts in the US have expressed their own frustrations when adjudicating and determining civil penalties or financial compensation for such human injuries such as "pain", "distress", "psychological trauma”, "suffering" as well as "emotional distress". These terms are illdefined and not codified in the laws, so even "plain language" terms such as these have confounded human juries and courts. In scientific research involving animals these Congressional mandates set the stage for institutional conflicts between regulatory agencies and between government and private industry that have lasted over 20 years.

Over the last 20 years of conducting research under the AWA the scientific, regulatory, and animal care communities still disagree with respect to a universally accepted operational or lexical definition of "distress". The National Research Council (NRC) has yet to support objective criteria or principles with which to qualify distress. The objective scientific assessment of subjective emotional states is yet to be proven and while there is often a measure of agreement on the interpretation of physiologic and/ or behavioral variables as indicators of stress, distress, or welfare status, there has not always a direct link. The NRC's position is that even if a universally accepted definition existed, it could not be applied across all species and all conditions, because of the differential impact of the strain, age, gender, genetic background and environment [4]. When drug approval agencies cannot yet agree on these statutory control issues, a "double bind" dilemma is imposed onto the pharmaceutical registrants under the administrative review process of these agencies. It is up to federal regulatory agency administrators to recognize this dilemma and take the appropriate steps to address this situation. While FDA, DEA, EPA, and USDA guidelines are not legally binding on the agencies, or their registrants, everyone involved in nonclinical research involving animals is legally bound under the law to follow the AWA.

As SDs conducting contractual research projects under the federally mandated "good laboratory practice" guidelines [2], it is oftentimes necessary to balance between:1) theexpectations and demands of our Sponsor, 2) the statutory or administrative requirements of multiple federal regulatory agencies (FDA, USDA, EPA, DEA, NIDA), 3) the general principals of nonregulatory agencies (PHS, NIH), as well as 4) other International commitments (International Council on Harmonization) to conduct sound science. These 4 major concerns each represents competing interests that must be kept in balance by the single point of control - the SD. If this is not difficult enough, as an employee of the CRO, the SD must consider the financial profit of the study, as well. All of this may sound like a reasonable expectation until the discipline of the science of the nonclinical research is identified as toxicology.

Toxicology is the study of adverse effects of xenobiotics on living systems and applies safety evaluations and risk assessments to the discipline. According to Eaton \& Klaasen [5] descriptive animal toxicity testing assumes that the effects produced by a compound in experimental animals are applicable to humans and that exposure of animals to toxic agents in high doses is a necessary and valid method of discovering possible hazards in humans.

Toxicology is the study of "poisons". It represents a methodical evaluation of the cellular, biochemical, and molecular mechanisms by which novel compounds exert adverse changes in living experimental subjects. It is the understanding that toxicology involves the intentional induction of test article-related effects (up to and including adverse effects and mortality) on living organisms and the ecosystems. These effects can include death with the intent of identifying the mechanisms of the toxicity and the precipitating causes and premonitory clinical signs of death.Federal authorities accept the fact that some performance measures in human patients and nonclinical animal studies will induce pain. In safety assessment testing conducted under legally binding performance requirements it is the SD's obligation to minimize the magnitude and duration of pain as well as alleviate unnecessary pain and distress of all experimental animals under their care whenever possible. Human research does not mitigate against nociceptive-inducing procedures, but it does mitigate the limitations posed on the intentional induction of pain in animals.

A SD must ensure "quality-based", valid, and reliable data that meet a minimal standard of regulatory acceptance with the assurance that such data were generated from living intact animals. These constraints effectively place the SD conducting 
Citation: David V. Gauvin, Lisa Craig and Scott E. Boley (2018) Statutory Imposed Terms of Stress, Distress, Well-Being and Animal Welfare: Suggested Guidelines for Humane Endpoints in Animal Studies. J.Pharm Pharm Scien 2(2): 1-13. doi: https://doi.org/10.24218/vjpps.2018.21.

the near-herculean balancing act while standing on a tight-rope 300 feet above the floor, without a safety net.

\section{The Guiding Principles of Research Conduct}

During the conduct of administering new drugs to animals, the subjective clinical judgments of technical staff are central to the evaluation of the animal's well-being and the veterinary clinical staff's request for euthanasia.One key term that is central to the basic concept of "humane endpoint" is "imminent death". Although the term "imminent death" is increasingly being used in recent human clinical literature with respect to organ donation, it is seldom defined in detail. The meaning of imminent death refers to the point of departure from active monitoring or medical treatment of a study animal to the recognition of potential organ or tissue damage that may compromise the completion of the study objectives required for regulatory review. With this in mind, each IACUC must develop a set of criteria that measures a realistic balance between scientific merit and animal welfare. This remains an open debate by the veterinary staff, SDs, sponsors and test facility management in CROs. The SD must meet the stated objectives of the regulatory-based study protocol whenever possible. In order to determine a cause of death certain biological or behavioral data may be required prior to scheduling or conducting euthanasia. These "value added" biomarkers must not compromise the ability for veterinary pathologists to process, interpret, and integrate histologically-based decisions to the study report in order to avoid repeating the study and using more animals (3R's - reduction).

Subjective evaluations may be biased when used to predict an animal's imminent death. Objective data-based approaches to predicting imminent death developed for specific experimental models could facilitate the implementation of timely euthanasia before the onset of clinically overt signs of moribundity and could thereby reduce pain and distress experienced by experimental animals.

Under Policy 11 of the AWA the Institutional Animal Care and Use Committee (IACUC) is required to ensure the minimization of pain and distress in animals during the conduct of bonafide research. This definition of painful procedure is direct from the regulations,

\section{A state in which an animal cannot escape from or adapt to the external or internal stressors or conditions that it experiences resulting in negative effects upon its well-being.}

Under the AWA, the SD is not given guidance as to the duration of "painful procedures" or the duration of time that must be provided to the animal in order to allow it to "adapt to the stressor"; however, it must be remembered that habituation, tolerance, and adaptation take time. The SD must establish the allowable time limit window to allow for these adaptations prior to early termination of the animal from the study.

Policy 12 of the AWA also calls forthe Institutional Animal Care and Use Committee (IACUC) to encourage and apply a two-fold test for protocol reviews in considering pain and distress. The first part of the test involves whether there is enough information for the IACUC to make a determination as to whether the SD has made a good faith and reasonable effort to determine what alternatives are available. The second part of the test focuses on whether adequate consideration was given to bona fide alternatives that were identified in the prescribed literature search.

The phrase "humane endpoint" was first legally recognized across the European Union (EU) on January 1, 2013 when member states were required to implement Directive 2010/63/EU [6] on the protection of animals used for scientific purposes. The term "humane endpoints" was first defined as,

"the earliest indicator in an animal experiment of severe pain, severe distress, suffering or impending death" [6].

More recently "humane endpoints" has been further described as "a refinement strategy designed to minimize pain, suffering or distress experienced by animals during an experiment" ([7], p. 344).

The inclusion of the term "distress" is for AWA purposes. No doubt there are other scenarios and situations where this definition of distress might not be appropriate, but it is common practice to limit "distress" to AWA purposes. Most laboratories define distress as those conditions that are in excess of that which an animal would experience by routine husbandry or handling practices. In other words, simply caging an animal in a laboratory is not to be considered distress in the context of the AWA and federal regulatory standards.

The International Association for the Study of Pain has defined the "pain" experience as, "an unpleasant sensory and emotional experience that is associated with actual or potential tissue damage or described in such terms....pain is always subjective" (https://www.iasp-pain.org/PublicationsNews/Content. aspx?ItemNumber=1673, but see [8]). As sentient, ethical human beings there is a natural tendency to observe an animal based on past experiences and to interpret and comment on what is perceived or believed to be the animal's status relative to discomfort, pain, or distress. It is very difficult, if not impossible, for past personal experiences to be meaningfully applied to an animal. Training and experience in studying and observing animal behavior are required to interpret and characterize that observation $[9,10]$. Pain is not a simple sensation, but rather a complex experience, only a part of which is sensory in nature. Accordingly, it is most accurate to describe what is studied as pain in animals as nociception, or the sensory nervous system's response to harmful, or potentially harmful stimuli [11-13]. Animals cannot express in words the psychological and emotional consequences of a noxious stimulus or event, however, most technicians and observers would not hesitate to apply the term "pain" to that circumstance. This fundamental distinction between pain and nociception emphasizes the importance of 
Citation: David V. Gauvin, Lisa Craig and Scott E. Boley (2018) Statutory Imposed Terms of Stress, Distress, Well-Being and Animal Welfare: Suggested Guidelines for Humane Endpoints in Animal Studies. J.Pharm Pharm Scien 2(2): 1-13. doi: https://doi.org/10.24218/vjpps.2018.21.

interpretation of animal behavior by an experienced individual to assess the presence and intensity of pain and distress.It is nevertheless imperative to acknowledge that novel compounds with unknown biological effects are being tested. Some animals are going to be adversely impacted, some may die.As described above, most laboratory workers have established the tenet that those procedures that produce pain in man might also produce pain in animals.

With respect to animal research, a distinction between stress and distress is critical in that the requirements for minimization of pain and distress apply to "distress" and not to "stress." Kathryn Bayne has proposed that:

1) Pain and distress are not discrete states, but are a continuum of experience;

2) Signs differ between species, and most animals hide signs of pain because such a sign of weakness may provoke an attack from predators or subordinate members of the group;

3) There is a lack of specific behavioral indicators of pain;

4) Inter-observer variability can be large; and

5) There is a tendency to anthropomorphize, which is encouraged by US Government

\section{Principle IV.}

\section{Principle IV of the AWA states that:}

Unless the contrary is established, investigators should consider that procedures that cause pain or distress in human beings may cause pain or distress in other animals.

The obligation to alleviate unnecessary pain and distress by the AWA is an important correlate of the responsible use of animals in biomedical research. Toxicology protocols can be associated with high mortality rates or require the production of progressive and severe clinical states that clearly could cause the deaths of experimental animals. These types of protocols or their associated Animal Care and Use Procedures (ACUPs) generally specify conditions under which preemptive euthanasia will be performed and may state that animals will be euthanized when they become "moribund" or are classified as "in extremis." However, SDs and Sponsors with diverse biomedical backgrounds may have varying concepts of the term's implications, rendering it poorly defined and operationally very difficult to interpret.

The lexical or dictionary definition of "moribund" include words and phrases such as "dying," "at the point of death," "in the state of dying," or "approaching death." However, these definitions are severely limited for laboratory animal research because they do not describe the moribund state in behavioral or physiological terms. Developing a sound approach to identifying the "moribund" or "in extremis" state is crucial to its effective use as a humane endpoint.

An objective, data-based approach to predicting death in the context of specific study designs or mechanisms of action should help to provide an unambiguous signal that an objectively verifiable endpoint has been reached thereby facilitating the IACUC approved process by which technical staff implement timely euthanasia to reduce any further pain and distress.

The termination of an experimental animal in a moribund state is preferred to "death as an experimental endpoint" because of the assumption that euthanizing a moribund animal will avoid or reduce terminal distress (see [14]). The problem in experimental laboratories is that the image triggered by the term "moribund" is often one of a prostrate, unresponsive, and perhaps seemingly comatose animal. From that perspective, one might seriously question whether such severely debilitated animals continue to experience pain or distress. If moribund animals are physiologically debilitated beyond the capacity for cognitive awareness of aversive sensations, euthanasia to avoid "spontaneous" death would not significantly reduce terminal distress.

Alternatively one can argue that, an unresponsive apparently moribund animal cannot necessarily be considered unaware, and one cannot necessarily assume that an unresponsive animal can no longer experience pain. The possibility that even unresponsive subjects might experience pain or distress reinforces the need for a clear, objectively-characterized definition of "moribund" or "in extremis" state that ensures:

1) The potential for suffering by the animals can be minimized by timely euthanasia,

2) All possible treatment options described in the protocol or ACUP have been implemented, and

3) Provides for the maximal amount of valid and reliable data to be collected that may help to elucidate the primary cause of the debilitative state of the animal that could influence further studies with the test article.

Test Facility Management and the IACUC have accepted the obligation to alleviate the unnecessary pain and distress of experimental animals and mandate the implementation of timely euthanasia. There is an acknowledgement of the tenuousness of an objectively-defined universal physiological endpoint for animals under a variety of study protocols. Also acknowledged is the fact that the background, education, and experience of both veterinarians and SDs in research laboratories may provide a subjective clinical judgment that is essential for the evaluation of the animal's well-being and support their mutual decision and prerogative to require activation of the "euthanasia for humane reasons" procedures. Objective, data-based approaches to predicting imminent death developed for specific experimental protocols may facilitate the implementation of timely euthanasia before the onset of clinically overt signs of moribundity and could thereby reduce pain and distress experienced by experimental animals in a nonclinical safety study. 
Citation: David V. Gauvin, Lisa Craig and Scott E. Boley (2018) Statutory Imposed Terms of Stress, Distress, Well-Being and Animal Welfare: Suggested Guidelines for Humane Endpoints in Animal Studies. J.Pharm Pharm Scien 2(2): 1-13. doi: https://doi.org/10.24218/vjpps.2018.21.

\section{The Goal of the SD}

The $\mathrm{SD}$ is required to establish definitions, operational checklists, or some other alternative to operationally define or characterize moribundity and "in extremis" prior to initiating the required studies. Toth [15] has highlighted the fact that persons with different biomedical backgrounds may have varying concepts of the term "moribund", rendering it poorly defined and arbitrarily interpreted once a study has started. Toth [15] also points out that these definitions are severely limited for laboratory animal research because they do not describe the moribund state in an objectively verifiable manner using behavioral or physiological terms. For a clear and generally accepted animal welfare policy, developing a sound approach to identifying the moribund state is crucial to its effective use as an experimental endpoint. The moribund condition typically implies a severely debilitated state that precedes imminent death.

The National Research Council's (US) committee on recognition and alleviation of pain in laboratory animals [4], the committee on regulatory issues in animal care [8], and the committee on the well-being of nonhuman primates [16] have repeatedly raised the question of when a study using animals should end, or the study design be changed due to animal pain, distress, or welfare considerations. Defining a humane endpoint can vary widely depending on a number of factors, of which study design and research objectives are but two. Consequently, attempting to provide specific endpoint criteria for all study designs or delineate other factors determinative of "distress" cannot be adequately addressed in any one document $[17,18]$. According to the NRC, defining specific endpoints not only would be inadequate, it could prove detrimental to unknown study objectives $[4,8,16]$. This exercise in due diligence does not go into specifics but rather presents selected points of interest that the SD, veterinarians, and the IACUC may use to open a dialogue in discussions prior to study protocol approvals. SDs, study personnel, veterinary staff, and IACUCs are obligated to thoroughly research and incorporate humane endpoints in every study involving laboratory animals.

It is imperative to have a set of agreed-upon terms that are used in the day-to-day management of animal studies that can be applied "across the board" for the majority of all studies conducted within a research facility.

Preemptive euthanasia could have several advantages for research:

Data collected after severe physiological impairments develop may not be useful or may be misleading for some purposes, and tissues that might otherwise be lost can be collected for postmortem analysis.However, in some cases these data may provide a significant interpretative value in determining the mechanisms of overt toxicity or cause of death;

If the Sponsor, SD, and operational technical staff recognizes the advantages of timely euthanasia when developing study endpoints, compliance with established endpoint criteria will undoubtedly be easier to achieve; and finally

A clear definition of the humane endpoints could also improve the ability of staff veterinarians and technicians to promote animal well-being and provide the most efficient collection of high-quality data.

Ashall\& Millar [19] have proposed several different types of experimental endpoints which are important for the articulation of "humane".Most laboratories and federal regulators have accepted that the concept of "unpredicted" should be considered alongside "scientific" and "justifiable" when determining critical decision-making endpoints, with each requiring individual consideration alongside an understanding of their interactions. Through the development of an endpoint matrix Ashall\& Millar [19] have provided a format for the consideration of each of these three types of endpoints, suggesting that:

a) Each type of endpoint is accurately defined;

b) The practical application of each endpoint to the experiment is determined; and

c) Information is collected which will allow endpoints to be accurately detected.

It is suggested that the further development and use of the endpoint matrix within each research institution may result in the appropriate selection of the most humane endpoint and should reduce overall animal suffering through more effective use of this refinement strategy.Hendricksen, et al [7] have proposed that there are four specific situations in which the application of humane endpoints are considered:

When the scientific objectives have been met and there is no reason to continue the investigation.

For example, in a dose range finding study when a maximum tolerated dose has been achieved.

When unexpected suffering occurs. In this situation, the suffering is not related to the experiment but is unexpected.

For example, in a respiratory function study in which "gasping" or unconsciousness is noted following dosing.

When suffering was anticipated at the start of the experiment, but has become more severe than predicted.

For example, in an efficacy study, using a rodent model of arthritis, and individual sensitivities to the chemical-induction procedure are noted that result in unrelenting vocalizations or immobility. And,

When pain and/or distress are an inherent part of the experiment and are anticipated at the outset.

For example, conducting a dose-range finding study for a small molecule and there is a need to establish a maximum tolerated dose.

Defining humane endpoints that are predictive of moribundity 
Citation: David V. Gauvin, Lisa Craig and Scott E. Boley (2018) Statutory Imposed Terms of Stress, Distress, Well-Being and Animal Welfare: Suggested Guidelines for Humane Endpoints in Animal Studies. J.Pharm Pharm Scien 2(2): 1-13. doi: https://doi.org/10.24218/vjpps.2018.21.

could have several advantages to the protocol objectives. On the one hand, animals would clearly benefit because unnecessary terminal distress could be eliminated or significantly reduced; and on the other hand, the final dataset might be more robust and allow experimental goals to be met more consistently. If one could accurately predict the time of death, euthanasia could be scheduled to permit timely collection of samples that could be lost if the animal died unexpectedly. Marczewski et al, [20] described a rat tumor study that targeted the presence of hind limb paralysis, rather than death, as an endpoint, thereby allowing the collection of tissues necessary for histopathologic documentation of the extent of the tumor and the possible response to therapy. In addition, an animal "in extremis" might modify important physiologic variables, rendering data collected under those conditions unusually variable or even uninterpretable within the context of the study.

Objective definition of the "moribundity" requires the differentiation of dying from illness, pain, and distress. But as admonished by Morton \& Griffiths [21], the decision to sacrifice the animals should not only depend on sum values, but mainly on the individual evaluation of each animal as a whole. An assessment of clinical and behavioral signs can certainly indicate that euthanasia is warranted for humane reasons, but many clinical signs, despite their severity, may not predict imminent or even eventual death. For example, Beagle dogs have been reported to express [22] spontaneous seizures.A seizure on study may indicate toxicity that is severe enough to warrant euthanasia for humane reasons, but obviously in many situations animals can live for a long time after having an ictal event, and indeed they may never have another seizure.

Pre-study scoring systems based on the severity of multiple behavioral or physiologic abnormalities [22,23] can provide operationally defined "benchmarks" for animal evaluations on study. These criteria can be used to support the research team's (SD, techs and veterinarians) determinations that euthanasia is warranted for humane reasons.Caution should be taken here, because there should be more than one endpoint since a single endpoint is not a valid or reliable instrument of measure for determining when, or if, euthanasia should be recommended.

For example, administering certain drugs, like barbiturates, benzodiazepines, alcohol, or anesthetics will induce hypothermia and an unresponsive state from which the animal will eventually recover. In this situation, these conditions obviously do not indicate a moribund state because the effects are due to the pharmacology of the drug. Second, the experimental endpoint can be modified as more is learned about the model. An example provided by Toth [15], is a report by Huang et al. $[24,25]$ studying mice inoculated with a myeloma cell line. In that study animals developed hindlimb paralysis, indicative of metastatic compression of the spinal cord, approximately 2 to 7 days before death. In a later follow up study, the experimental endpoint was redefined, and hind limb paralysis was used as an indicator for preemptive euthanasia [24,25]. As experience with and data collected from a specific predictive model are collected, more information will be available for developing endpoint refinements.

Another example admonishing the use of a single endpoint selection for pre-emptive euthanasia is body temperature. According to Toth [15], hypothermia is perhaps the most commonly reported predictor of experimental animals' imminent death [26-30]. Its use as an endpoint requires determination of a specific index temperature or temperature range that is invariably associated with imminent death. Preemptive euthanasia is then performed if an animal's temperature drops below the $a$ priori, mutually agreed upon, core body temperature. Soothill, et al [28], for example, reported mice with acute experimental bacterial infections developed rectal temperatures of less than $34^{\circ} \mathrm{C}$ before the onset of clinically overt toxicity that eventually warranted euthanasia. In studies of influenza-infected mice, rectal temperatures of less than $32^{\circ} \mathrm{C}$ were inevitably associated with death in one study [30], whereas another found that mice recovered after even more profound hypothermia and used a core temperature of $28^{\circ} \mathrm{C}$ as the indication for euthanasia [31]. In a general toxicity study, Gordon, et al [26] found a linear relationship between the $50 \%$ lethal dose and the dose of metallic salts that reduced body temperature to $35^{\circ} \mathrm{C}$. Premorbid variability in temperature, uninterrupted wheel-running for $3 \mathrm{hrs}$, and hypothermia below $30^{\circ} \mathrm{C}$ were sequential temporal markers of moribundity and death in rats studied in an activitystress model [32].

The research team should be aware of relevant issues related to the singular use of temperature as a critical sign of imminent death:

(1) Body temperature can be significantly influenced by ambient temperature and by other aspects of the environment, such as the type of bedding or the presence of cage mates. This consideration is particularly important for mice. Thus, selection criteria developed under one experimental situation may not be applicable in all situations.

(2) Different strains or species of animals may react differently to the same challenge. For example, $\mathrm{BALB} / \mathrm{c}$ mice are more sensitive than $\mathrm{C} 57 \mathrm{BL} / 6$ mice to influenza challenge, demonstrating higher mortality rates [31] and perhaps a different critical index temperature [33]. In contrast, staphylococcal enterotoxin A elicits more severe hypothermia and greater rates of mortality in $\mathrm{C} 57 \mathrm{BL} / 6$ mice than in $\mathrm{BALB} / \mathrm{c}$ mice [29].

(3) The method of temperature measurement may influence the recorded values. Radiotelemetery provides a continuous record of core temperatures and can be used to evaluate the duration of hypothermia [31], but data collection with this type of system requires expensive equipment and surgical manipulation of the animal and is rarely used in toxicology 
Citation: David V. Gauvin, Lisa Craig and Scott E. Boley (2018) Statutory Imposed Terms of Stress, Distress, Well-Being and Animal Welfare: Suggested Guidelines for Humane Endpoints in Animal Studies. J.Pharm Pharm Scien 2(2): 1-13. doi: https://doi.org/10.24218/vjpps.2018.21.

study designs. Implanted microchips and rectal measurements using hand-held thermometers or probes provide only a snapshot evaluation of temperature that may be 1 to $1.5^{\circ} \mathrm{C}$ different from actual core temperatures as a result of animal restraint and stress. In some cases, prolonged hypothermia may reflect imminent death, whereas transient or short-term hypothermia may resolve and be associated with eventual recovery.

As described previously the AWA defines distress as: A state in which an animal cannot escape from, or adapt to, the external or internal stressors or conditions that it experiences resulting in negative effects upon its well-being. If these animals were euthanized prematurely, then the failure to show that the animals cannot adapt or recover from hypothermia induced by a given study design or test article does not comply with the intent of the AWA or the objectives of conducting the study in the first place. Premature euthanasia may require more animals to be added the study violating the 3R's "reduction" of animals use required by the AWA.

Another simple but specific endpoint marker that is visually obvious, objective, and easy to assess is the inability to rise or ambulate. This condition was a good predictor of imminent death in guinea pigs with Pseudomonas-induced sepsis [34] and in mice with endotoxemia[35]. Moreover, mice with severe or palpable hypothermia are also likely to be recumbent and unresponsive to handling or other stimuli [35]. In contrast, many rodent studies conducted with ethyl alcohol report lethargy, ataxia, sedation, and hypothermia following doses of 2 to $4 \mathrm{~g}$ / $\mathrm{kg}$ that fully recover within 12 to 14 hours [36,37]. Tolerance develops to these behaviorally disruptive effects of ethyl alcohol. Premature euthanasia would prevent the often-times required demonstration of tolerance development on the study plan [38].

\section{As Single Point of Control, the SD Must Set the Stage}

It is imperative that each SD establishes a conversation with the Sponsor, in-house veterinary staff, IACUC, and supportive operational staff with respect to how the study is going to be conducted. The conversation should cover the limitations, expectations, and even the frustrations that are associated with a specific study protocol. These discussions will be influenced by the study type and be different if conducting a dose-range finding study, a 3-month repeat-dose study, or a 2 year carcinogenicity study. An outcome of these discussions should be to develop some endpoints or targets for interventions by veterinary staff. As detailed by Toth [15] as a starting point these include:

\section{Scientific Enpoint}

The scientific endpoint is defined as "the criteria that will be used to indicate that the experimental objective has been reached" [39]. This endpoint relates to the data which must be gathered to achieve the objectives of an experiment. Under GLPs of the FDA [2] the scientific objectives of the study must be clearly defined in the protocol prior to IACUC approval.
Once the study objective is met further scientific information is not required, and any suffering (whether severe or not) caused if an experiment continues beyond this point would be considered "avoidable." Using the objective of the study as an endpoint is humane because this represents the earliest point at which the experiment can be ended and still achieve the goal of the study. However, an experiment will only be ethically acceptable within a cost/benefit calculus when the scientific endpoint proposed falls within the limits of permissible suffering. As first detailed by Toth [15] and expanded by Trammell \&Toth [40] these previous reports are used as a starting point here, to include.

\section{Justifiable endpoint}

The identification of "humane endpoints" through cost/benefit justification in animal experiments has been addressed following the drafting of Directive 2010/63/EU by Lindl, et al [41]. The IACUC must determine a justifiable balance between the needs of humans, animals or the environment and the pain, suffering, and distress of animals proposed to meet these needs. Lindl, et al [41] have proposed the use of an original term, i.e., "justifiable endpoint," to define humane endpoints which are based upon a cost/benefit justification.

As part of the IACUC approval of experiments expected to cause any pain or suffering, the benefits expected to be achieved must be used to justify a specified level of suffering. The justifiable endpoint is reached once this level of pain or suffering is detected. As it is not considered ethically justifiable to exceed this endpoint, it could be considered the absolute limit of "humane" experimentation. Hendriksen, et al [7] have highlighted the important interaction between the scientific endpoint and a humane endpoint identified during cost/benefit justification: if the scientific endpoint cannot be reliably reached before the justifiable endpoint, the recommendation would be that animals be removed from the study before the experimental aims are achieved, resulting in data loss and therefore unjustifiable animal use.

It is clear that these two humane endpoints do not encompass a clear consideration of any unexpected outcomes that may occur on a study. Unexpected suffering can occur during a study that may be due to changes which are not directly related to the protocol requirements or to suffering which is different from that expected as a result of the assays required by the protocol. These "unpredicted endpoints", which may not have been previously defined by the Sponsor or SD, or predicted by the background, education, and experience of the members of the IACUC (institutional memory) should be considered and initiate a conversation to establish a veterinary action plan prior to starting the study .

\section{Unpredicted endpoint}

Unexpected suffering might occur following an unrelated illness or accident or from unexpected adverse effects of the test article. The IACUC and SD should understand that unexpected suffering 
Citation: David V. Gauvin, Lisa Craig and Scott E. Boley (2018) Statutory Imposed Terms of Stress, Distress, Well-Being and Animal Welfare: Suggested Guidelines for Humane Endpoints in Animal Studies. J.Pharm Pharm Scien 2(2): 1-13. doi: https://doi.org/10.24218/vjpps.2018.21.

might necessitate humane intervention, irrespective of whether the scientific or justifiable endpoint has been reached. Some unpredicted adverse events may occur due to the development of sensitization, such as:

1. skin abrasions due to over grooming (stereotypy)

2. scratching/barbering as a result of pruritis

3. rodent bumblefoot (ulcerative pododermatitis)

4. over aggression to cagemates

5. spontaneous mammary tumors

Since the suffering is not related to the attainment of the scientific goal or has not been factored into a cost-benefit justification for the study, this type of animal welfare issue presents a greater challenge to the IACUC since its' form and severity cannot be factored into the cost-benefit justification during research planning. By definition the "unpredicted endpoint" cannot be defined in advance; however, the knowledge that unpredicted events do occur on studies should initiate a general discussion as to the role of the Sponsor in contemporaneous decision making. That is, can the SD act on behalf of the sponsor in times when rapid decisions are called for?

It is critical to the general animal welfare policy of every research institution that the management of unpredicted endpoints is carefully considered so a veterinary medical intervention plan is defined as part of the protocol development process or part of a CROs SOPs. When expected suffering occurs it is presumed that a veterinary consult has already been requested and the technical staff should be regularly documenting any additional adverse events that may be occurring. Since the level of suffering justified by the expected benefits of the experiment should not be exceeded, the concept of "cumulative suffering" becomes essential and critical. This concept has previously been applied to cumulative measurements of the severity or duration of suffering in experimental animals [42]. A discussion on an action plan and the possibility of "early termination" should be initiated if the suffering caused by adverse events alone or in addition to any expected suffering exceeds that which was justified by the expected benefits of the study and initially approved by the IACUC.

In practice this would mean that animals in distress due to unpredicted adverse events should not simultaneously be exposed to the further levels of expected suffering justified in the cost/benefit analysis. Unless adverse events can be managed under the clinical guidance of the staff veterinarian that alleviates or mitigates the suffering OR if the scientific endpoint can be achieved well before the identified justifiable endpoint, adverse events might therefore lead to humane intervention before the scientific endpoint is achieved. To address this scenario, Ashall\& Millar [19] have proposed a detailed decision matrix that can be used to assist the research team in formulating a response in such situations.

\section{While Central Authority Resides in the SD, Others are Involved in the Process}

The Office of Laboratory Animal Welfare (OLAW) within the National Institutes of Health (NIH) has defined the "[criteria used to end experimental studies earlier in order to avoid or terminate unrelieved pain and/or distress are referred to as humane endpoints". As previously discussed, an important feature of humane endpoints is that they should ensure that study objectives will still be met even though the study is ended at an earlier point than the scheduled study termination date. Ideally, humane endpoints are sought that can be used to end studies before the onset of pain and distress" ([43], page 103). In 1994, the OECD recognized that while ambiguous test guidelines may be necessary, such ambiguity fosters an overbroad interpretation of what constitutes a humane endpoint in toxicology studies. The US is not bound by the OECD initiatives. However, the OECD created a working group to develop a guidance document using clinical signs as humane endpoints in safety evaluation studies [44] (Table, below):

The resulting document put forth criteria based on the principles of the 3Rs as well as descriptions of clinical signs to assist study personnel in determining when death may be imminent or when

Table: OECD Guidance Document on the Recognition, Assessment, and Use of Clinical Signs as Humane Endpoints for Experimental Animals Used in Safety Evaluation (OECD 2000)

A humane endpoint can be defined as the earliest indicator in an animal experiment of severe pain, severe distress, suffering, or impending death.

The ultimate purpose of the application of humane endpoints to toxicology studies is to be able to accurately predict severe pain, severe distress, suffering, or impending death, before the animal experiences these effects. However, the science of toxicology is not yet to the point where such accurate predictions can be made prior to the onset of severe pain and distress. It is possible at this time to identify pain, distress, or suffering, very early after their onset by careful clinical examination of animals on test using well-defined endpoints and criteria. Humane endpoints for use in research and testing have been addressed in a number of publications. . . . These adverse conditions, once identified, should be minimized or eliminated, either by humanely killing the animal or, in long-term studies, by (temporary) termination of exposure, or by reduction of the test substance dose.

Different animal species, and animals at different stages of development, may respond differently to test conditions, and exhibit different indications of distress. The clinical signs described here should be evaluated in consideration of these potential differences. If relevant humane endpoints have been identified, they should be described when an experiment is being planned, and incorporated into the experimental protocol and all related standard operating procedures (SOPs). 
Citation: David V. Gauvin, Lisa Craig and Scott E. Boley (2018) Statutory Imposed Terms of Stress, Distress, Well-Being and Animal Welfare: Suggested Guidelines for Humane Endpoints in Animal Studies. J.Pharm Pharm Scien 2(2): 1-13. doi: https://doi.org/10.24218/vjpps.2018.21.

severe pain may be present after an animal's exposure to a test substance. The criteria are broad enough to apply to a wide range of study types, test substances, species, and strains of animals. The reader is encouraged to examine this resource when developing internal guidance documents to assess humane endpoints.

In the US, humane endpoints must be reliable, reproducible, and objective, and allow both the achievement of study objectives and the use of appropriate methodologies at the earliest point to alleviate or avoid pain. The NRC acknowledges that pilot studies are an effective means to identify and validate humane endpoints, which can then be incorporated into subsequent studies to minimize, alleviate, or avoid pain for the animal subjects (also see $[17,18]$; and [4], p. 61).

The NRC acknowledges the difficulties in developing an institutional or global policy on humane endpoints. The IACUC realizes the emotional impact involved in study decisions regarding pain and distress. The AWA [1] also acknowledges that toxicity studies will induce pain and distress under some conditions, but it is the goal to reduce such pain and distress whenever possible. While the ideal is to avoid pain, personnel also need to work towards meeting the study objectives before an animal is euthanized [43]. Under US regulatory control, three specific points of interest must be kept in mind in developing humane endpoints:

If a full study, or aspect of a study, is ended before the objectives have been met, one can argue that the animals used have been wasted.

Moreover, if the purpose of a study is to evaluate the toxicity of the test article as part of the safety assessment, a regulatory agency may reject the submitted data as insufficient and require that the study be repeated.

If researchers are reluctant to intervene, study animals may experience unnecessary pain, distress, or severely diminished welfare.

Without adequate IACUC guidance, death is likely to be selected as a convenient endpoint that is reproducible and objective. Note that if regulatory guidelines do not specify an endpoint, as in vaccine potency studies [45], regulated entities can and will use lethality.OLAW has approached the subject of "humane endpoints" in its IACUC Guidebook [46] advising the IACUC to review protocols to determine whether

discomfort to animals will be limited to that which is unavoidable for the conduct of scientifically valuable research, and [whether] unrelieved pain and distress will only continue for the duration necessary to accomplish the scientific objectives.

The OLAW reference is careful to acknowledge potential pain or distress in animal studies, however these should be relieved with appropriate medication, or with euthanasia, BUT the study objectives should still be met whenever possible.

\section{SD Defines Humane Endpoints in Toxicology Studies}

The U.S. Environmental Protection Agency Health Effects Test Guidelines for Acute Oral Toxicity $[47,48]$ provide instruction for following the OECD Guidance Document [49] to reduce the suffering of animals in toxicity studies. Euthanasia of animals that are either moribund or in severe pain is also encouraged. Regrettably, vague statements such as "animals showing severe and enduring signs of distress and pain may need to be humanely killed," which are common in regulatory guidelines, may promote a reluctance to terminate a study or an animal's exposure to the testing substance because a regulatory body may consider the action premature and mandate a repeat study. This is not a good situation for researchers, laboratories, or animals.

In 2004 the US government required animal ocular irritancy testing prior to market approval. For the first time the government published a notice in the Federal Register in 2007 from the Interagency Coordinating Committee on the Validation of Alternative Methods (ICCVAM) to request the submission of data and information on the use of topical anesthetics and systemic analgesics for alleviating pain and distress in rabbits during eye irritation testing (Federal Register, May 9, 2007; Volume 72, Issue 89: p. 26396). These data would be used to evaluate whether the use of topical anesthetics and/or analgesics to reduce pain and distress for in vivo testing situations could compromise the study objectives. Current guidelines include neither justification for withholding analgesic agents nor guidance for the use of analgesic agents to alleviate ongoing pain. As a result, testing entities may be reluctant to provide analgesia beyond initial local anesthetics, to avoid the possibility of interference with the test substance. Numerous published studies have demonstrated that the use of analgesics to alleviate pain from ocular irritancy tests do not interfere with the scientific objectives of this safety test $[29,50,51]$. Such evidence can be used in the protocol or ACUP for ocular irritancy studies to avoid or alleviate pain as well as to provide scientific rationale for the use of analgesics in these tests.

Carcinogenicity testing are currently required to assess the safety of chronically administered drugs by exposing rodents to the test article for their lifetime. The incidence of tumor burden, geriatric changes, and premature death can be significant, particularly near the scheduled termination of these studies. Guidelines generally specify the survival rates necessary to provide meaningful interpretation of a carcinogenicity study. The OECD is the only source of material to discuss "humane endpoints" and provide guidance for the early termination of a study if survival rates fall below a specified percentage. It is tempting for SDs to adopt a "no-euthanasia policy" to achieve the required survival rate at the end of the study. In doing so, animals often are not euthanized until very close to death, an outcome that may entail needless pain for the animals. This approach would be perceived to be in accordance with the 3R's because the regulatory agency may decide that sufficient numbers of animals did not complete the 
Citation: David V. Gauvin, Lisa Craig and Scott E. Boley (2018) Statutory Imposed Terms of Stress, Distress, Well-Being and Animal Welfare: Suggested Guidelines for Humane Endpoints in Animal Studies. J.Pharm Pharm Scien 2(2): 1-13. doi: https://doi.org/10.24218/vjpps.2018.21.

study and the data have insufficient statistical power for critical safety assessment determination and require another study.

One of the sources reviewed for the NRC report was the approach developed by the Health and Environmental Sciences Institute (HESI) of the International Life Sciences Institute [52]. In 2000, this organization redesigned safety testing schemes for agricultural chemicals. The resulting multifaceted approach was adopted by the International Commission on Harmonisation (ICH) and the US FDA.In these harmonized study guidelines traditional toxicology tests were redesigned to integrate several sciences, such as metabolism/kinetics and life stages, in a single study to eliminate the requirement for separate studies to evaluate each parameter and reduce the number of animals used [52-55]. Further, the metabolism/kinetics component of the strategy can be particularly relevant to the alleviation of pain in laboratory animals: based on the metabolism of a test substance in the animal model. This approach, based on step-wise, or tiered testing, helps to reduce animal numbers, minimize potential pain to laboratory animals by avoiding exposure levels that produce clinical signs of toxicity, and improve the quality of data used in human risk assessments [53].

The IACUC does also acknowledge that in some isolated instances, guidelines require that a certain percentage of control animals die before a test is considered valid, while other tests are based on the survival of the vaccinated animals, however this is a small percentage of animals studies conducted. For example, the FDAadministered safety test for general biological products requires vaccination of healthy guinea pigs and mice with a small dose of the final product from each vaccine lot (21CFR, Ch. 13: 2008, $\$ 610.11)$. A safety test is considered unsatisfactory if the animals do not survive the 7-day test period, in which case additional safety tests over a larger test population are required. The USDAmandated potency testing for Leptospirapomonabacterin (CFR $2006, \$ 113.101)$ requires that at least eight of ten unvaccinated control animals die in order to validate the test.

Of critical importance to improvements in quality of life for both humans and animals, is research on pain itself, including the mechanisms of pain and methods of pain alleviation. Complicating the ethical issues inherent in producing pain in research subjects is the ability to accurately predict and measure pain responses in the subjects. It is imperative for pain investigators to establish endpoints in each study design to minimize the duration and intensity of the pain and to validate those endpoints for the integrity, objectivity, and reproducibility of the study. A productive dialogue between the IACUC, staff veterinarians and the SD is critical for ensuring the best outcome for both the animals' welfare and the study objectives in the study.

A humane death, or endpoint, is a fundamental tenet of the US Principles for the Utilization and Care of Vertebrate Animals Used in Testing, Research, and Training[46], as Principle VI states that "[a] nimals that would otherwise suffer severe or chronic pain that cannot be relieved should be painlessly killed at the end of the procedure or, if appropriate, during the procedure." There is no rigidly defined point at which euthanasia should be performed for humane reasons, as it is not possible to apply a single set of euthanasia criteria across all study designs, animal models, and experimental goals. The decision should involve a team approach among veterinarians, SDs, and animal care personnel using all available information about the affected animal(s). Body condition scores can be used to determine when to consider euthanasia for humane reasons. The earliest possible indicators for euthanasia should be clearly identified so as to avoid pain and yet still achieve study objectives.

Identification and validation of humane endpoints should be considered for studies involving pain, but this is neither an easy nor a simple process:

1. It is important to ensure that endpoints are validated and based on sound science.

2. Given the wide scope of procedures and goals of animal research, no single reference can document every humane endpoint for every research protocol. Given the variety involved in nonclinical safety assessment, a weight of evidence approach should be taken to define when euthanasia should be considered that is tailored to the study type, test article pharmacology, and clinical indication.Good communication between SDs, veterinary staff, animal care staff, and the IACUC is crucial.

3. Efforts should continue in the development and validation of alternative procedures for incorporation into toxicology studies to avoid or alleviate pain in laboratory animals.

\section{Suggested Guidelines for Humane Endpoints in Animal Studies}

Based on the collective work of the published reports appearing in peer reviewed scientific journals described, above, and the cumulative IACUC experiences of the three authors here are some guidelines that may initiate the conversation between $\mathrm{SD}$, Client, and veterinary staff at a test facility.

1) Each Animal Care \& Use Protocol (ACUP), especially those that are anticipated to result in severe or chronic pain, should describe endpoint(s) and specify a plan and criteria for removal/euthanasia of animals from the study, or the disposition of animals at the termination of the study. For many studies, the endpoint will be euthanasia upon study completion, euthanasia at certain time points, or the return of animals to stock. For studies where moderate to severe clinical signs can be anticipated, the endpoint description in the ACUP should include identification of personnel responsible for decision making, specific criteria (body weight, mass size, appetite, etc.) that will be monitored at prescribed frequencies (daily, weekly, etc), and a disposition (treatment, euthanasia, early removal from study, etc.) once those criteria have been met or exceeded. 
Citation: David V. Gauvin, Lisa Craig and Scott E. Boley (2018) Statutory Imposed Terms of Stress, Distress, Well-Being and Animal Welfare: Suggested Guidelines for Humane Endpoints in Animal Studies. J.Pharm Pharm Scien 2(2): 1-13. doi: https://doi.org/10.24218/vjpps.2018.21.

2) Animal studies may involve procedures that cause severe clinical signs or morbidity, and investigators should consider the selection of the most appropriate endpoint(s) for their study. This requires careful consideration of the scientific objectives of the study, the expected and possible adverse effects the research animals may experience, the most likely time course and progression of those adverse effects, and the earliest most predictive indicators of present or impending adverse effects. Prior to the initiation of the study, the SD should determine the criteria that would lead to termination of the study for any animal, when appropriate, and the method of euthanasia to be employed.

3) A clear chain of command for the decision-making process should be documented, including contingency plans if said individuals are unavailable for consultation. This should be done prior to the start of dosing. Optimally, studies are terminated when animals begin to exhibit severe clinical signs if this endpoint is compatible with meeting the research objectives. Such endpoints are preferable to death or moribundity as endpoints since they minimize pain and distress.

4) If available, there should be scientific justification in the ACUP for allowing an animal to die without intervention if the goals of a study can be accomplished by euthanizing animals before they become moribund.

5) Animals involved in experiments that may lead to moribundity or death should be monitored daily (including weekends) by personnel experienced in recognizing signs of morbidity. Once severe clinical signs develop, more frequent observation (2-3 times daily) may be required.

Based on experience and background published data, the following conditions may warrant early termination. The SD should provide scientific justification for exemptions:

Rapid (within one week) weight loss of $\geq 20 \%$ of body weight

1a) Unless this is a biomarker for the objectives of the study - for example, drug dependence protocols;morphine withdrawal in rats will induce 20 to 30 grams of bodyweight per day over the first 3 to 5 days following the abrupt cessation of opiate administrations).

2) Extended period of weight loss, progressing to a dramatically thin and physically weak state.

3) Surgical complications unresponsive to medical intervention.

4) Combination of the following: poor physical appearance (very rough hair coat, abnormal posture, labored breathing); abnormal behavior (reduced mobility/unconsciousness, unsolicited vocalizations, self-mutilation); severe depression or abnormal/exaggerated responses to external stimuli. 4a. Except in cases in which presentation of these clinical signs are biomarkers required to be documented as objectives of the study protocol (for example, drug withdrawal syndromes induced by positive control articles).

5) Severe respiratory distress or depression that does not show adaptation/ tolerance/ habituation over time (tachyphlaxis) or which is unresponsive to treatment.

6) Occurrence of a serious injury or trauma from which recovery is unlikely.

7) Neurological signs: persistent convulsions, persistent stereotypies that interfere with the activities of daily living (i.e., eating, drinking, over the established fasting duration limits of standard SOPs (i.e. 16 hours); or motor impairments that interfere with eating and drinking that extend longer than the allowable fasting duration limits and from which recovery is unlikely.

8) Excessive bleeding from any orifice, which is unresponsive to treatment.

9) One or more skin ulcers that do not heal, depending upon the species and severity of the ulcers.

10) Mass size or location that interferes with normal function or ulcerates with no evidence of healing.

11) A mass that is estimated by size to be greater than $15 \%$ of normal body weight.

12) For carcinogenicity studies it is necessary to rely on experience and good judgment when deciding when to euthanize an animal as a result of one or more masses. Many of these masses grow slowly and do not compromise the animal.

\section{Conclusion}

The $\mathrm{SD}$ is responsible for ensuring that the federal regulatory policies of the USDA, FDA, EPA, and OECD are followed. Exceptions to this policy should be scientifically justified and approved by the IACUC before they can be implemented.In spite of the centralized control of study functions given to the SD under the FDA GLPs, it is the IACUC that has the ultimate authority, mandated by law [1], to act on behalf of the Test Facility Management and Institutional Officer (IO) to investigate and, if necessary, suspend any activity which violates applicable laws, regulations, standards, guidelines, policies and procedures.

\section{Acknowledgement}

This review represents a work product for MPI Research.No outside funding was dispersed and all 3 authors were actively employed during the manuscript devilment process. There are no conflicts of interest from any author. 
Citation: David V. Gauvin, Lisa Craig and Scott E. Boley (2018) Statutory Imposed Terms of Stress, Distress, Well-Being and Animal Welfare: Suggested Guidelines for Humane Endpoints in Animal Studies. J.Pharm Pharm Scien 2(2): 1-13. doi: https://doi.org/10.24218/vjpps.2018.21.

\section{References}

1. United States Department of Agriculture. Animal Welfare Act. United States Code, Title 7 (Agriculture), Chapter 54 (Transportation,Sale, and Handling of Certain Animals), 1996; Sections 2131-2159.

2. United States Food and Drug Administration.Good Laboratory Practices for Nonclinical Laboratory Studies. Title 21--Food And Drugs, Chapter I--Food And Drug Administration, Department Of Health And Human Services, Subchapter A-General, Part 58 Good Laboratory Practice For Nonclinical Laboratory Studies; 1978. Available from:https://www.accessdata.fda.gov/scripts/ cdrh/cfdocs/cfcfr/cfrsearch.cfm?cfrpart=58.

3. United States Congress. Public Law 111-274.Plain Writing Act of 2010. 5 USC §301. Available from:https://www.gpo.gov/fdsys/ pkg/PLAW-111publ274/pdf/PLAW-111publ274.pdf.

4. National Research Council. Committee on recognition and alleviation of pain in laboratory animals.Recognition and alleviation of pain in laboratory animals.Washington DC: The National Academies Press; 2009.

5. Eaton DLL, Klaassen CD. Principles of Toxicology. In: CD Klaassen, JB Watkins III, editors.Casarett and Doull's: Essentials of Toxicology. New York: McGraw-Hill; 6-20 p.

6. The European Parliament And The Council Of The European Union. Directive 2010/63/EuOf The European Parliament And Of The Council of 2010 Sep 22. On the protection of animals used for scientific purposes. 2010. Available from:http://eur-lex.europa. eu/legal-content/EN/TXT/?uri=celex\%3A32010L0063.

7. Hendriksen $C$, Morton D, Cussler, K. Use of humane end points to minimise suffering. In: B Howard, T Nevalainen, G Perretta, editors. The cost manual of laboratory animal care and use.Boca Raton, FL: CRC Press; 2011.333-353 p.

8. National Research Council. Committee on regulatory issues in animal care and use. Definition of pain and distress and reporting requirements for laboratory animals. Proceedings of the workshop held 2000 Jun 22. Washington DC: National Academy Press; 2000.

9. Gebhart GF. Visceral pain-peripheral sensitization. Gut. 2000; 47(Suppl 4):iv54-iv55.

10. Gebhart GF. J.J. Bonica Lecture--2000: Physiology, pathophysiology, and pharmacology of visceral pain. RegAnesth Pain Med. 2000; 25(6):632-638.

11. Morgan MM, Fossum EN, Stalding BM, King MM. Morphine antinociceptive potency on chemical, mechanical, and thermal nociceptive tests in the rat. J Pain. 2006; 7(5):358-366.

12. Sneddon LU. Pain perception in fish: indicators and endpoints. ILAR J. 2009; 50(4):338-342.

13. Crook RJ, Walters ET. Nociceptive behavior and physiology of molluscs: animal welfare implications. ILAR J. 2011; 52(2):185195.

14. Gauvin DV, Zimmermann ZJ, Baird TJ. Further support for the AVMA-approved rodent euthanasia protocol. J Anim Res Vet Sci. 1: 002.

15. Toth LA. Defining the moribund condition as an experimental endpoint for animal research. ILAR J. 2000; 41(2):72-79.

16. National Research Council.Committee on well-being of nonhuman primates. The psychological well-being of nonhuman primates. Washington DC: National Academy Press; 1998.
17. Morton DB. Humane endpoints in animal experimentation for biomedical research: Ethical, legal and practical aspects. In: CFM Hendriksen, DB Morton, editors. Humane Endpoints in Animal Experimentation for Biomedical Research. London: Royal Society of Medicine Press, 1999.5-12 p.

18. Morton DB. A systematic approach for establishing humane endpoints. ILAR J. 2000; 41(2): 80-86.

19. Ashall V, Millar K. Endpoint matrix: a conceptual tool to promote consideration of the multiple dimensions of humane endpoints. ALTEX. 2014; 31(2):209-213. doi: 10.14573/altex.1307261.

20. Marczewski K, Halliop J, Dziemidok P, Korczak E, ObuchowskaPrzebirowska D, Tochman A, et al. Does moxonidine increase cytotoxic activity of ethanol in rats? Preliminary report on the base of the ultrastructural study. Arch ImmunolTherExp (Warsz). 1998; 46(4):241-246.

21. Morton DB, Griffiths PH. Guidelines on the recognition of pain, distress and discomfort in experimental animals and an hypothesis for assessment. Vet Rec. 1985; 116(16):431-436.

22. Edmonds HL Jr, Hegreberg GA, vanGelder NM, Sylvester DM, Clemmons RM, Chatburn CG.Spontaneous convulsions in beagle dogs. Fed Proc. 1979; 38(10):2424-2428.

23. Olfert ED. Defining an acceptable end point in invasive experiments. Anim Welf Inf Ctr News lett. 1995; 6:3-7.

24. Huang YW, Richardson JA, Tong AW, Zhang BQ, Stone MJ, Vitetta ES. Disseminated growth of a human multiple myeloma cell line in mice with severe combined immunodeficiency disease. Cancer Res. 1993; 53(6):1392-1396

25. Huang YW, Richardson JA, Vitetta ES. Anti-CD54 (ICAM-1) has antitumor activity in SCID mice with human myeloma cells. Cancer Res. 1995; 55(3):610-616.

26. Gordon CJ, Fogelson L, Highfill JW. Hypothermia and hypometabolism: Sensitive indices of whole-body toxicity following exposure to metallic salts in the mouse. J Toxicol Environ Health. 1990; 29(2):185-200.

27. Kort WJ, Hekking-Weijma JM, TenKate MT, Sorm V. A microchip implant system as a method to determine body temperature of terminally ill rats and mice. Lab Anim. 1997; 32(3):260-269.

28. Soothill JS, Morton DB, Ahmad A. The HID ${ }_{50}$ (hypothermia-inducing dose 50): An alternative to the LD50 for measurement of bacterial virulence. Int J Exp Path. 1992; 73(1):95-98.

29. Stiles BG, Campbell YG, Castle RM, Grove SA. Correlation of temperature and toxicity in murine studies of staphylococcal enterotoxins and toxic shock syndrome toxin 1. Infect Immun. 1999; 67(3):1521-1525.

30. Wong $\mathrm{M}-\mathrm{L}$, Bongiorno $\mathrm{PB}$, Rettori $\mathrm{V}$, McCann SM, Licinio J. Interleukin (IL) 16, IL-1 receptor antagonist, IL-10, and IL-13 gene expression in the central nervous system and anterior pituitary during systemic inflammation: Pathophysiological implications. ProcNatlAcadSci U S A. 1997; 94(1):227-232.

31. Toth LA, Rehg JE, Webster RG. Strain differences in sleep and other pathophysiological sequelae of influenza virus infection in naive and immunized mice. J Neuroimmunol. 1995; 58(1):89-99.

32. Morrow NS, Schall M, Grijalva CV, Geiselman PJ, Garrick T, Nuccion $S$, et al. Body temperature and wheel running predict survival times in rats exposed to activity-stress. PhysiolBehav. 1997; 62(4):815-825 
Citation: David V. Gauvin, Lisa Craig and Scott E. Boley (2018) Statutory Imposed Terms of Stress, Distress, Well-Being and Animal Welfare: Suggested Guidelines for Humane Endpoints in Animal Studies. J.Pharm Pharm Scien 2(2): 1-13. doi: https://doi.org/10.24218/vjpps.2018.21.

33. Ray MA, Johnston NA,Verhulst S, Trammell RA, Toth LA. Identification of Markers for Imminent Death in Mice used in Longevity and Aging Research. J Am Assoc Lab Anim Sci. 2010; 49(3):282-288.

34. Louie A, Liu W, Liu Q-F, Sucke AC, Miller DA, Battles A, et al. Predictive values of several signs of infection as surrogate markers for mortality in a neutropenic guinea pig model of Pseudomonas aeruginosa sepsis. Lab Anim Sci. 1997; 47(6):617-623.

35. Krarup A, Chattopadhyay P, Bhattacharjee AK, Burge JR, Ruble $G R$. Evaluation of surrogate markers of impending death in the galactosamine-sensitized murine model of bacterial endotoxemia. Lab Anim Sci. 1999; 49(5):545-550.

36. Gauvin DV, Cheng EY, Holloway FA.Biobehavioral correlates of alcohol hangover In: Galanter M, editor.Recent Developments in Alcoholism: Ten Years of Progress. New York: Plenum Press; 1993.281-304p.

37. Gauvin DV, Goulden KL, Holloway FA.State dependent operant stimulus control: Cueing attributes of ethanol hangover in rats. Alcohol ClinExper Res. 1993; 17:1210-1214.

38. United States Food and Drug Administration, Center for Drug Evaluation and Research (CDER), Controlled Substances Staff (CSS), Assessment of Abuse Potential of Drugs Guidance for Industry January 2017. Available from:https://www.fda.gov/downloads/ drugs/guidancecomplianceregulatoryinformation/guidances/ ucm198650.pdf.

39. Wallace J. Humane endpoints and cancer research. ILAR J. 2000; 41(2):87-93.

40. Trammell RA, Toth LA. Markers for predicting death as an outcome for mice used in infectious disease research. Comp Med. 2011; 61: 492-498.

41. Lindl T, Gross U, Ruhdel I, von Aulock S, Völkel M. Guidance on determining indispensibility and balancing potential benefits of animal experiments with costs to the animals with specific consideration of EU Directive 2010/63/EU. ALTEX. 2012; 29(2):219228.

42. Honess $\mathrm{P}$, Wolfensohn $\mathrm{S}$. The extended welfare assessment grid: A matrix for the assessment of welfare and cumulative suffering in experimental animals. Altern Lab Anim. 2010; 38(3):205-212.

43. United States National Institutes of Health. Office of Laboratory Animal Welfare (OLAW). Applied Research Ethics National Association (ARENA). Institutional Animal Care and Use Committee Guidebook. 2 ${ }^{\text {nd }} e d ; 2002$.

44. Organisation for Economic Co-operation and Development (OECD). OECD Environmental Health and Safety Publications Series on Testing and Assessment.No. 19. Guidance Document On The Recognition, Assessment, And Use Of Clinical Signs As Humane Endpoints For Experimental Animals Used In Safety Evaluation; 2000.
45. United States Department of Agriculture.Animal Welfare Regulations. Code of Federal Regulations, Title 9 (Animals and Animal Products), Chapter 1 (Animal and Plant Health Inspection Service, Department of Agriculture), Subchapter A (Animal Welfare), Parts 1-4].

46. United States Department of Health and Human Services, National Institutes of Health Office of Laboratory Animal Welfare Public Health Service Policy on Humane Care and Use of Laboratory Animals. Public Health Service Policy on Humane Care and Use of Laboratory Animals; 2015.

47. United States Environmental Protection Agency (EPA). Federal Insecticide, Fungicide, and Rodenticide Act (FIFRA). Good Laboratory Practice Standards. Final Rule. 40 CFR Part 160. Washington DC: GPO; 1998.

48. United States Environmental Protection Agency (EPA). Health effects test guidelines. Washington DC: Office of Pesticides, Prevention, and Toxic Substances. Available from:http://www. epa.gov/docs.OPPTS_Harmonized/870_Health_Effects_Test_ Guidelines/Series/.

49. European Union (EU). Directive 2010/63/EU of the European Parliament and of the Council of 22 September 2010 on the protection of animals used for scientific purposes. Official J EU L. 2010; 276:33-79. Available from http://eur.lex.europa.eu/ LexUriServ/LexUriServ.do?uri=OJ:L:2010:276:0033:0079:en:PDF

50. Patrone G, Sacca SC, Macri A, Rolando M. Evaluation of the analgesic effect of $0.1 \%$ indomethacin solution on corneal abrasions. Ophthalmologica. 1999; 213(6):350-354.

51. Peyman GA, Rahimy MH, Fernandes ML. Effects of morphine on corneal sensitivity and epithelial wound healing: implications for topical ophthalmic analgesia. Br J Ophthalmol. 1994;78(2):138141.

52. Bass AS, Darpo B, Breidenbach A, Bruse K, Feldman HS, Garnes D, et al. International Life Sciences Institute (Health and Environmental Sciences Institute, HESI) initiative on moving towards better predictors of drug-induced torsades de pointes. $\mathrm{Br} J$ Pharmacol. 2008; 154(7):1491-1501.

53. Carmichael DA, Down MP, Shillcock RC, Eagleman DM, Simner J. Validating a standardised test battery for synesthesia: Does the Synesthesia Battery reliably detect synesthesia? Conscious Cogn. 2015; 33:375-85

54. Cooper RL, Lamb JC, Barlow SM, Bentley K, Brady AM, Doerrer NG, et al. A tiered approach to life stages testing for agricultural chemical safety assessment. Crit Rev Toxicol. 2006; 36(1):69-98.

55. Doe JE, Boobis AR, Blacker A, Dellarco V, Doerrer NG, Franklin C, et al. A tiered approach to systemic toxicity testing for agricultural chemical Safety Assessment. Crit Rev Toxicol. 2006; 36(1):37-68. 